\title{
Treatment of Penile Prosthesis Implant's Infection
}

\author{
Marco Cosentino $^{a}$ Marta Bianco $^{b}$ Eduard Ruiz-Castañéc Massimo lafrate ${ }^{b}$ \\ ${ }^{a}$ Head of Andrology and Urology Department, Casa di Cura Villa Maria, Padova, Italy; ${ }^{b}$ Department of Surgical, \\ Oncological and Gastroenterological Sciences, Urology Clinic, University of Padua, Padua, Italy; ' ${ }^{\mathrm{H}}$ ead of Andrology \\ Department, Fundació Puigvert, Universitat Autònoma de Barcelona, Barcelona, Spain
}

\section{Keywords}

Prosthesis infection - Penile prosthesis implant · Infection .

Prosthesis explant · Treatment infection

\begin{abstract}
Introduction: Penile prosthesis implant is a safe and effective option in erectile dysfunction patients, being implant procedures safe with a low risk of infection. However, when infection occurs, it represents a concrete problem for both surgeon and patient. Methods: This is a comprehensive review of all issues relating to prosthesis infection, including causes and risk factors, methods of prevention, and management. We analyzed all preoperative and perioperative factors, which can play a role in infection of the device. Results: Infection of penile prosthesis implant is hard to manage and correct. While the incidence of infection following first implant is up to $3 \%$, in cases of re-implant surgery, the rate can reach as high as $18 \%$. Many articles were found addressing prevention and treatment of penile prosthesis infection, and many analyzed all relevant pre- and perioperative factors associated with penile prosthesis implant. Although such factors have been well studied, there is no clear consensus worldwide on certain topics. Conclusions: Penile prosthesis implant is a safe and effective option. Despite infection is a rare event, surgeons should follow strictly pre-, intra- and postoperative recommendations in order to reduce the risk
\end{abstract}

of device's infection. An appropriate antibiotic therapy should be tailored on patient's characteristics and pathogens isolated.

(c) 2020 S. Karger AG, Basel

\section{Introduction}

Erectile dysfunction is defined as the inability to have and/or sustain an erection sufficient for a sexual intercourse and affects more than 150 million men across the world $[1,2]$. Penile prosthesis implant is a safe and effective option in patients with erectile dysfunction when all other options are not working (lifestyle changes, PDE-5, intra-cavernosal injection, vacuum and device). Surgical implant procedures are now safe surgeries with excellent results; reliability and durability of the prostheses have improved, although malfunction and infection represent a high percentage of complications. Especially, penile prosthesis infection (PPI) represents one of the worst complication of andrologic surgery; it is associated with morbidity for patients and with a high healthcare cost

Part of this article was published first as a chapter in the Living Handbook on "Urogenital Infections and Inflammations" edited by the European Association of Urology, Arnhem, The Netherlands. Published as Open-Access Publication by GMS gGmbH, Germany.

karger@karger.com

www.karger.com/uin

(c) 2020 S. Karger AG, Basel

Karger ${ }^{\prime}=$
Marco Cosentino

Casa di Cura Villa Maria, Head of Andrology and Urology Department Via delle Melette 20

IT-35138 Padova (Italy)

doccosentino@gmail.com 
that exceeds the cost of the first implant by more than 6 -fold $[3,4]$. Aim of this study is to identify all possible causes of prosthesis infection focusing on which are the main pathogens involved and pathway of contamination, methods of prevention, and management of prosthesis infection.

\section{Materials and Methods}

A systematic review of the literature was performed over the past 20 years using search terms including: inflatable penile prosthesis, penile implantation, infection, prevention of infection, and antibiotic therapy. We considered all articles that addressed infectious complications in patients undergoing PPI surgery, with emphasis on causes, detection, diagnosis, and management. Only articles in English were considered.

\section{Results and Discussion}

We can divide the risk factors into related to the patients (i.e., diabetes mellitus, obesity, immunosuppression) and related to the procedure (i.e., revision surgery, antibiotic prosthesis coating, previous or concomitant surgeries). Among all the risk factors, the most important ones seem to be, without doubt, the presence of diabetes mellitus and the reintervention (defined as a change of penile prosthesis): in fact, while the incidence of infection after de novo implant is found only in $1-3 \%$ of the patients, in cases of revision surgery, the risk increases to $10 \%$ and in diabetic patients, it has even been reported to reach $18 \%$ [5]. Many of the selected articles addressed the prevention of PPI, but there is no clear consensus worldwide on certain topics, including the use of antibiotic therapy: about this topic, the 2 most relevant studies $[6,7]$ suggested that higher doses of gentamicin act as a protective factor; however, no significant association was found.

The study by Gross et al. [7, 8] also identifies the most implicated pathogens: they obtained 227 intraoperative cultures at salvage or explantation and identified 204 organisms and documented a high incidence of infections with Escherichia coli (18.3\%), coagulase-negative Staphylococcus (15\%), Candida species (11.1\%), MSSA (10.5\%), and MRSA (9.2\%). Intravenous antibiotic regimens for all patients at implantation were generally, but not always, consistent with AUA or EAU guidelines: 22\% (49 of 227) received a cephalosporin (cefazolin) and an aminoglycoside (gentamicin) and 56\% (126 of 227) received vancomycin and an aminoglycoside (gentamicin) at implantation.

Penile Prosthesis Implant Infection
Table 1. Microorganism identified and agent utilized

\begin{tabular}{ll}
\hline Microorganism & Therapeutic agents \\
\hline S. epidermidis & Gentamycin/ampicillin \\
S. aureus & Vancomycin \\
Group B Streptococcus & Ampicillin/gentamycin \\
E. coli & Third-generation cephalosporins \\
Pseudomonas aeruginosa & Piperacillin-tazobactam \\
Candida & Fluconazole \\
\hline
\end{tabular}

Indeed, the AUA recommends an aminoglycoside (or aztreonam in patients with renal compromise) in combination with a first- or second-generation cephalosporin or vancomycin. The combination of an aminoglycoside (or aztreonam) and vancomycin showed the greatest efficacy, eliminating $86 \%$ (175 of 204) of the microbes found in culture in our series. However, this combination had poor anaerobic coverage $(25 \%, 4$ of 16$)$ and lacked fungal coverage.

The EAU suggests a second- or third-generation cephalosporin or a penicillin agent with anti-penicillinase efficacy. Ampicillin-sulbactam was the most effective single anti-penicillinase agent in the EAU guidelines and eliminated $72 \%$ (146 of 204) of the cultured microbes in our series. The anaerobic coverage was excellent (100\%, 16 of 16) but sacrificed Gram-positive and Gram-negative coverage (72 and 73\%, respectively), did not cover Candida species, and was not used by our surgeons. In conclusion, the microorganisms identified in these studies were not covered by the AUA and EAU antibiotic guidelines in at least $14-38 \%$ of cases. These findings suggest broadening antibiotic prophylaxis guidelines and creating a management algorithm for IPP infections might lower infection rates and improve salvage success (Table 1). Based on the results, in another study, Gross et al. [8] specifically examined the patients affected by Candida and found that most were diabetic and overweight and the majority of them were also first-time implant recipients. The study was limited by a low sample size (26 patients) but represents the largest population of patients with fungal PPI reported to date and, according to the authors, additional antifungal prophylaxis may be warranted in diabetic and obese patients, although further investigation is needed. In order to prevent PPI by inhibiting prosthesis microbial attachment, the 2 main penile prosthesis manufacturers use a hydrophobic coating for devices in order to create a physical barrier. The use of antibiotic-impregnated implants was found by Carson et al. [9] to reduce 
the rate of initial revision due to PPI from 2.5\% (when using nonimpregnated implants) to $1.1 \%$ at 7.7 years of follow-up [9]. Also, a review performed by Mandava et al. [10] found that the infection-retardant coatings decrease the incidence of device infection by approximately $50 \%$. Further technological advancements for the prevention of biofilm formation are warranted, and other speculative methods have recently been discussed in an article by $\mathrm{He}-$ rati and Lo [11]. Hematoma is another complication associated with an increased risk of infection. It usually presents in the early postoperative period, with an incidence ranging from 0.2 to $3.6 \%$ [12]. Wrapping of the genitalia for a compressive dressing and closed-suction drainage is associated with a statistically significant reduction in the rate of hematoma formation to $0.9 \%$ [13$15]$. We can find many studies about expedients to prevent infection, especially regarding the preoperative and peri/intraoperative measures; however, studies are lacking concerning in particular the postoperative measures to be followed by patients once at home after discharge. It is commonly believed that some trivial measures like adequate hygiene, local application of ice, and the use of suspensory or wrap-around underwear may help to reduce the risk of PPI. A recent review by Hebert and Kohler [16] examined multiple preoperative/intraoperative factors evaluating their influence on the risk of PPI and divided them into elements that increase the risk, elements that reduce the risk, and elements that do not influence the risk. They used the 2011 Oxford Center for EvidenceBased Medicine Guidelines to give each conclusion a level of evidence (from Level 1 that means strongest evidence to Level 5 that represents weaker evidence). Multiple factors were associated with increased risk of post-prosthesis placement infection, including smoking tobacco (Level 1), CD4 T-cell count $<300$ (Level 4), Staphylococcus aureus nasal carriage (Level 2), revision surgery (Level 2), prior spinal cord injury (Level 3), and hemoglobin A1c level $>8.5$ (Level 2). Factors with no effect on infection rate included preoperative cleansing with antiseptic (Level 4), history of prior radiation (Level 3), history of urinary diversion (Level 4), obesity (Level 3), concomitant circumcision (Level 3), immunosuppression (Level 4), age $>75$ (Level 4), type of hand cleansing (Level 1), postsurgical drain placement (Level 3), and surgical approach (Level 4). Factors associated with decreased rates of infection included surgeon experience (Level 2), "No Touch" technique (Level 3), preoperative parenteral antibiotics (Level 2), antibiotic-coated devices (Level 2), and operative field hair removal with clippers (Level 1). Among the protective factors mentioned there are the "No Touch" technique and hair removal with the use of clippers, proving that the main source of contamination is the perineal skins. As for the removal of preoperative body hair, it is usually preformed with a clipper or razor. Due to skin folds, scrotal skin poses a difficult surface for hair removal. Multiple studies have addressed methods of hair removal and an SSI risk. In a Cochrane review of 6 trials addressing hair removal before surgery versus no hair removal, no statistically significant difference was found in rate of postoperative infection. However, use of a razor for hair removal compared to use of clippers was associated with an increase in postoperative infection (relative risk $=2.09,95 \%$ confidence interval $=1.15-3.80)$. It is also important to notice that, although the 2016 ICSM guidelines recommend the use of an alcohol-based skin prep prior to IPP placement [16], the studies we found are discordant and did not show the superiority of a disinfectant on the others $[17,18]$. Further research is needed into the standardization of pre-, peri-, and postoperative measures: guidance on these measures needs to be clear to both surgeons and patients. PPI can present in 2 different ways: a relatively silent form with local symptoms that is due to Staphylococcus epidermidis (up to $80 \%$ of cases) or other coagulase-negative bacteria and an "aggressive" form with systemic effects that is due to E. coli, S. aureus, Klebsiella, Serratia, or Pseudomonas [19]. Both forms can require removal of the entire device. One of the most problems of the conservative treatments is the presence of the biofilm, which impedes antibiotic penetration and reduces phagocytosis $[20,21]$. Once established which is the microorganism involved in the infection, an appropriate antibiotic therapy should be promptly performed (Table 1).

Furthermore, a conservative management of the infected prosthesis is very difficult to achieve and not every patient is eligible for that: the decision to conserve a prosthesis or not depend on the patient's presentation and clinical status, the timeline and onset of infection, and response to antibiotic treatment. Possible failure of conservative treatment should be weighed against urgent explantation to avoid sepsis and glans or shaft necrosis. Various conditions, including cylinder migration, erosion of the corpora cavernosa or corpus spongiosum, and sepsis in a patient with comorbidities such as uncontrolled diabetes, require revision surgery and the prosthesis removal as soon as possible. For several reasons, including technical ones, many propose the implantation of a new prosthesis at the time of explant surgery ("concomitant implant after explant"). A penis uninhabited by the cylinder will lead to fibrosis formation and shortening of the 
penis (by up to $4-5 \mathrm{~cm}$ ) [22]; in these circumstances, reimplant surgery is technically demanding, and the use of a vacuum device after explant may help in obtaining good results and reducing fibrosis and the shortening of the penis. Although technically harder, delayed re-implant surgery currently remains safer than immediate re-implant surgery. While newer salvage techniques have demonstrated increased success [21], further strong studies are required to identify how to improve techniques and strategies for immediate salvage procedures, enabling a complete exploitation of their benefits relative to delayed re-implantation, cost effectiveness, technically easier, and with a reduced risk of penis shortening.

\section{Conclusions}

Penile prosthesis implant is a safe procedure with a relatively low risk of infection in the case of a first implant. When infection does occur, prosthesis explant may be required, with distress for the patient and the need for further complex surgery with an attendant-increased healthcare cost. There are various risk factors for infection and while many of them have been well studied, PPI still occurs. An appropriated antibiotic therapy should be tailored on patient's characteristics and type of microorganism infection.

\section{Disclosure Statement}

No conflict of interest or financial support for this paper.

\section{Author Contributions}

Marco Cosentino: conception and design, drafting, data acquisition, and final approval. Massimo Iafrate: drafting and revision. Marta Bianco: data acquisition and revision. Eduard Ruiz Castane: revision and final approval.

\section{References}

$1 \mathrm{NIH}$ consensus conference. Impotence. NIH consensus development panel on impotence. JAMA. 1993;270(1):83-90.

2 Lee DJ, Najari BB, Davison WL, Al Hussein Al Awamlh B, Zhao F, Paduch DA, et al. Trends in the utilization of penile prostheses in the treatment of erectile dysfunction in the United States. J Sex Med. 2015;12(7):1638-45.

3 Balen A, Gross MS, Phillips EA, Henry GD, Munarriz R. Active polysubstance abuse concurrent with surgery as a possible newly identified infection risk factor in inflatable penile prosthesis placement based on a retrospective analysis of health and socioeconomic factors. J Sex Med. 2016;13(4):697-701.

4 Carson CC. Diagnosis, treatment and prevention of penile prosthesis infection. Int J Impot Res. 2003;15(Suppl 5):S139-46.

5 Carvajal A, Benavides J, García-Perdomo H, Henry G. Risk factors associated with penile prosthesis infection: systematic review and meta-analysis. Int J Impot Res. Feb 2020. https://doi.org/10.1038/s41443-020-0232-x.

6 Xie D, Gheiler V, Lopez I, Nehrenz GM, Klopukh B, Bianco F, et al. Experience with prophylactic gentamicin during penile prosthesis surgery: a retrospective comparison of two different doses. J Sex Med. 2017; 14(9):1160-4.

7 Gross MS, Phillips EA, Carrasquillo RJ, Thornton A, Greenfield JM, Levine LA, et al. Multicenter investigation of the micro-organisms involved in penile prosthesis infection: an analysis of the efficacy of the AUA and EAU guidelines for penile prosthesis prophylaxis. J Sex Med. 2017;14(3):455-63.
8 Gross MS, Reinstatler L, Henry GD, Honig SC, Stahl PJ, Burnett AL, et al. Multicenter investigation of fungal infections of inflatable penile prostheses. J Sex Med. 2019 Jul; 16(7):1100-5.

9 Carson CC, Mulcahy JJ, Harsch MR. Longterm infection outcomes after original antibiotic impregnated inflatable penile prosthesis implants: up to 7.7 years of followup. J Urol. 2011;185(2):614-8.

10 Mandava SH, Serefoglu EC, Freier MT, Wilson SK, Hellstrom WJ. Infection retardant coated inflatable penile prostheses decrease the incidence of infection: a systematic review and meta-analysis. J Urol. 2012 Nov; 188(5):1855-60.

11 Herati AS, Lo EM. Penile prosthesis biofilm formation and emerging therapies against them. Transl Androl Urol. 2018;7(6):960-7.

12 O’Rourke TK, Erbella A, Zhang Y, et al. Prevention, identification, and management of post-operative penile implant complications of infection, hematoma, and device malfunction. Transl Androl Urol. 2017;5:832-48.

13 Henry GD, Wilson SK. Updates in inflatable penile prostheses. Urol Clin North Am. 2007; 34(4);535-47.

14 Henry GD. The Henry mummy wrap and the Henry finger sweep surgical techniques. J Sex Med. 2009;6(3):619-22.
15 Wilson SK, Henry GD. Hematoma formation following penile prosthesis implantation: to drain or not to drain? J Urol. 1996;55:643A.

16 Hebert KJ, Kohler TS. Penile prosthesis infection: myths and realities. World J Mens Health. 2019 Sep;37(3):276-87.

17 Chlebicki MP, Safdar N, O’Horo JC, Maki DG. Preoperative chlorhexidine shower or bath for prevention of surgical site infection: a meta-analysis. Am J Infect Control. 2013; 41(2):167-73.

18 Webster J, Osborne S. Preoperative bathing or showering with skin antiseptics to prevent surgical site infection. Cochrane Database Syst Rev. 2015;(2):CD004985. https://doi. org/10.1002/14651858.CD004985.pub5.

19 Blum MD. Infection of genitourinary prosthesis. Infect Dis Clin. 1989;3:259-74.

20 Lotan Y, Roehrborn CG, McConnell JD, Hendin BN. Factors influencing the outcomes of penile prosthesis surgery at a teaching institution. Urology. 2003;62(5):918-21.

21 Krzastek SC, Smith R. An update on the best approaches to prevent complications in penile prosthesis recipients. Ther Adv Urol. 2019;11:1-9.

22 Martinez DR, Mennie PA, Carrion R. Erectile function significant enough for penetration during sexual intercourse after removal of inflatable penile prosthesis. J Sex Med. 2012; 9(11):2938-42. 